\title{
Awareness problems following moderate to severe traumatic brain injury: Prevalence, assessment methods, and injury correlates
}

\author{
Rodney D. Vanderploeg, PhD; ${ }^{1-4 *}$ Heather G. Belanger, PhD; ${ }^{1-3}$ Jennifer D. Duchnick, PhD; ${ }^{1}$ Glenn Curtiss ${ }^{1-2,4}$ \\ ${ }^{1}$ James A. Haley Veterans' Hospital, Tampa, FL; ${ }^{2}$ Defense and Veterans Brain Injury Center, Tampa, FL; Depart- \\ ments of ${ }^{3}$ Psychology and ${ }^{4}$ Psychiatry, University of South Florida, Tampa, FL
}

\begin{abstract}
We examined the degree to which individuals with a history of moderate to severe traumatic brain injury (TBI) are unaware of their postinjury deficits. We also examined correlates between awareness problems and injury acuteness and severity and whether awareness problems differed across behavioral domains. Self- and significant other (family member) ratings on the Key Behaviors Change Inventory (KBCI) were examined in 36 individuals an average of 2 years postTBI. Family members reported greater problems postinjury than patients did, depending on the behavior in question and the level of patient awareness overall. Postinjury awareness problems were as prevalent as other behavioral problems measured by the KBCI but were not universally present. Some patients exhibited no awareness problems, others emergent awareness, and a minority poor awareness. Correlations revealed that as time postinjury increased, patients showed more accurate self-awareness than those whose injuries were more recent. Family members and patients agreed about preinjury functioning.
\end{abstract}

Key words: awareness, brain injuries, Glasgow Coma Scale, neurobehavioral manifestations, postinjury deficits, posttraumatic amnesia, rehabilitation, self-awareness, TBI, unawareness.

\section{INTRODUCTION}

Individuals with traumatic brain injury (TBI) often have difficulty accurately perceiving changes in their level of functioning postinjury [1]. Unawareness of deficits contributes to problems across life areas, including return to productive employment [2] and maintenance of close relationships [2-4]. Of relevance to rehabilitation efforts, a patient who is unaware of deficits is less likely to actively participate in treatment or may refuse to participate altogether. For patients with more severe cognitive or behavioral deficits, unawareness can pose serious safety concerns.

Although patients with TBI who demonstrate unawareness of deficits, or anosognosia, typically have normal intelligence [5-6], they may manifest a variety of cognitive impairments during neuropsychological evaluation, including impaired memory ability [7-8], compromised executive functioning [9-10], and decreased fine motor speed [11]. Discrepancies have been noted, however, with some studies suggesting little to no relationship between level of awareness and performance on

\footnotetext{
Abbreviations: $\mathrm{ANOVA}=$ analysis of variance, $\mathrm{AQ}=$ Awareness Questionnaire, DVBIC $=$ Defense and Veterans Brain Injury Center, GCS $=$ Glasgow Coma Scale, GDS = global discrepancy score, GOAT $=$ Galveston Orientation and Amnesia Test, IRB $=$ institutional review board, $\mathrm{KBCI}=$ Key Behaviors Change Inventory, MS = multiple sclerosis, PCRS-NR = Patient Competency Rating Scale for Neurorehabilition, PTA = posttraumatic amnesia, $\mathrm{SD}=$ standard deviation, $\mathrm{TBI}=$ traumatic brain injury, VA $=$ Department of Veterans Affairs, VAMC $=$ VA medical center.

*Address all correspondence to Rodney D. Vanderploeg, PhD; Mental Health and Behavioral Sciences, Psychology, James A. Haley Veterans' Hospital, 13000 N. Bruce B. Downs Blvd, 116B, Tampa, FL 33612; 813-972-2000, ext 6728; fax: 813-903-4814. Email: Rodney.Vanderploeg@va.gov DOI: $10.1682 / J R R D .2006 .12 .0163$
} 
other cognitive measures [11-13]. This disparity has often been attributed to different methods of awareness assessment [12,14-15], although variance across studies in the injury severity and acuteness of participants could also be the cause.

Several approaches are used for assessing degree of awareness. The most common approach is an interview or checklist-based comparison of discrepancies between patient self-ratings of their postinjury problems and ratings by others (family members or treatment staff). The larger the discrepancy (other rater reporting greater problems than the individual with TBI), the larger the patient's underlying self-awareness deficit. Another method is comparing patients' self-ratings with their neuropsychological performance. The rationale is that patients with unawareness should demonstrate primarily executive dysfunction because of limited self-monitoring ability [9]. A third method is structured or semistructured interviews, such as the Self-Awareness of Deficits Interview [16], which involves an examiner asking the patient specific questions, with family members verifying the answers. The assessment is then scored according to a preestablished rating scale. A final method of assessment is rating actual behaviors that indicate unawareness on outcome measures such as the Key Behaviors Change Inventory (KBCI) [17]. Ratings typically are completed by family members. Other direct rating scales of unawareness used in TBI are the Patient Competency Rating Scale for Neurorehabilition (PCRS-NR) [18] and the Awareness Questionnaire (AQ) [19]. In a recent comparison of the latter two instruments, the original PCRSNR and the AQ were only modestly correlated, although they both predicted employability equally well from patient-clinician discrepancies [20].

In terms of self-other discrepancy ratings, mixed results have been found in the literature regarding degree of unawareness following TBI. For example, in individuals with primarily moderate to severe TBI, Cavallo and colleagues found that 39 percent of their patient and family member pairs were in high agreement about the patient's functioning 1 to 3 years postinjury on the Problem Checklist of the New York University Head Injury Family Interview [21]. High agreement was defined as agreement on the presence or absence of at least 75 percent of 34 common post-TBI problems. For 26 percent of the patient and family member pairs, the patient endorsed twice as many problems as the family member, and for 35 percent of the pairs, the family member endorsed twice as many problems as the patient. No differences were found for demographic variables or injury severity between these groups.

Seel and colleagues reported generally good postinjury agreement between injured individuals and family members using the Neurobehavioral Functioning Inventory, an instrument that contains 70 items and assesses specific behaviors and symptoms across six scalesdepression, somatic, memory/attention, communication, aggression, and motor [22]. Comparisons were made between patient and family member pairs an average of 2.2 years postinjury (median $=0.8$ years). Individuals with mild head injuries comprised more than half the sample. Paired $t$-tests revealed no significant differences between individuals' and families' responses on five of the six scales. Differences were found on the communication scale, and surprisingly, individuals with TBI reported more frequent problems than their family members did. Pagulayan et al., in a sample of patients with complicated mild to severe TBI, also found generally good agreement between individuals with TBI and their significant others on the Sickness Impact Profile, a 136item health status questionnaire that assesses sleep, emotional behavior, mobility, social interaction, etc. [23].

Others using self-other rating discrepancies have suggested that self-awareness in individuals with TBI is much more limited, with no significant agreement between the patient and the significant other. Specifically, Dywan and Segalowitz found nonsignificant correlations across five scales (planning, initiation, attention/ memory, arousal/inhibition, and social monitoring) between family members and patients with moderate to severe TBI an average of 10 years postinjury [24]. In addition, family members rated the patients more impaired in all domains except attention/memory. Similarly, in a study of individuals with a range of mild to severe TBI who were pursuing compensation litigation an average of 30 months postinjury, clinical interview data indicated that individuals post-TBI reported fewer cognitive-behavioral and emotional problems than their family members did [25]. No differences, however, were noted in the number of physical complaints. Of note, these interview data were obtained in an open-ended fashion with a nonstandardized interview.

Finally, some studies have found that concordance between individuals' and families' ratings is initially poor but improves with time postinjury [12,26]. Powell and colleagues, for instance, used an interview format in 
which patients were asked to give a number between 1 and 100 to indicate how close they were to being fully recovered and found that perceptions of recovery showed good relationships with the Glasgow Outcome Scale after 1 month [26]. Also, patients tended to report more cognitive problems over time, which suggests an inverse relationship between unawareness and chronicity. Similarly, Lanham and colleagues found increased concordance between patient and family member ratings on the Katz Adjustment Scale from 6 to 12 months postinjury in their sample of patients with moderate to severe TBI [12].

The degree of anosognosia found may depend in part on the outcome measure. For example, do self-other rating discrepancies have criterion validity? The limitation of the self-other discrepancy approach is assuming the "other" is accurate. As Fleming and colleagues pointed out, various factors influence significant others' perceptions of their loved one, including stress levels, personality of the relative, and length of time postinjury [16]. To the extent that family members are not objective, selfother rating discrepancies will not be accurate measures of unawareness.

The extent to which any of these methods is valid may also depend on the degree to which the target behavior is describable (concrete) versus nonspecific, generalized, and inferential. In general, concrete behaviors are easier to quantify. For example, physical and basic self-care activities typically do not result in rating discrepancies between patients and family members [25,27]. Sander and colleagues found good to "almost perfect" concordance on the Community Integration Questionnaire, which contains very concrete functional items [28].

Finally, using only one measurement method may be problematic given the potentially multidimensional nature of the construct. For instance, O'Keefe et al. recently demonstrated a distinction between metacognitive knowledge and more "online" types of awareness, such as emergent and anticipatory awareness [29]. This study highlighted the complexity of the awareness construct and the need to examine it with multiple methods.

In summary, the bulk of evidence in the literature supports the contention that patients with TBI, primarily those with moderate to severe injuries, have significant problems with awareness. The extent of this unawareness depends on many factors, which have undoubtedly contributed to some of the disparate findings. Some of these factors include sample characteristics (i.e., severity and chronicity), assessment method, and focus of the aware- ness assessment (i.e., patients are unaware of what?). Those studies that found good agreement between selfother ratings had methodological limitations [24-25] or primarily included participants with mild injuries [22].

Our first objective was to replicate and extend previous research by examining the specific degree to which individuals with a history of moderate to severe TBI in the chronic phase are unaware of deficits in cognitive, behavioral, and emotional domains - domains typically affected by brain injury. We used two methods of awareness assessment, self-other discrepancy ratings and a direct behavioral measure of awareness, to further clarify the degree of awareness problems in these patients. Our second objective was to examine the extent to which awareness of deficits and self-other rating agreement depends on the behavioral domain in question (e.g., somatic versus cognitive). Our third objective was to investigate relationships between awareness and indices of injury severity and acuteness. We hypothesized that more severe and acute injuries would be associated with a greater degree of unawareness. We used the KBCI, an instrument developed to assess changes in specific behaviors following TBI, to compare self-other ratings of functioning several years postinjury.

\section{METHODS}

\section{Participants}

Participants were active duty military personnel or veterans (gulf war era) who were enrolled in an institutional review board (IRB)-approved rehabilitation treatment trial conducted through the Defense and Veterans Brain Injury Center (DVBIC). The measures and methodology in this study were approved by the IRB. The DVBIC is a collaborative treatment and research project between the Department of Defense and the Department of Veterans Affairs (VA). As part of the treatment trial, all participants had completed an acute inpatient rehabilitation treatment program for TBI at the Tampa VA medical center (VAMC) and 1-year follow-up evaluations. Ninety-six individuals were eligible and were contacted either at the time of acute rehabilitation discharge or between 1 and 6 years after their inpatient rehabilitation treatment stay. Of the 96 patients, 3 refused to participate. Of the remaining 93, 62 participated but provided only partial data, for an overall response rate of 65 percent. A total of 36 postinjury individual-other pairs (38\%) 
returned complete data (self- and other ratings for preand postinjury functioning) and were included in this study. We found no differences on any demographic, injury, or acute rehabilitation treatment characteristics between the 36 individual-other pairs who participated in the study and the 60 who did not.

Participants had to have had a TBI confirmed by documentation in the medical record, subsequent posttraumatic amnesia (PTA) of $\geq 1$ day, and traumatic changes of the brain on computed tomography or magnetic resonance imaging to be included in the study. No participant had a history of prior TBI or other severe neurological or psychiatric condition (e.g., psychosis).

\section{Participants with TBI}

Participants with TBI were almost exclusively male (94\%), had a mean \pm standard deviation (SD) age of 38.9 years, and had a mean \pm SD education of $12.9 \pm$ 2.0 years. Most were injured in motor vehicle accidents $(79 \%)$ or falls $(12 \%)$. Initial emergency room Glasgow Coma Scale (GCS) [30] scores ranged from 3 to 14, with a mean $\pm \mathrm{SD}$ of $5.9 \pm 3.0($ median $=5.0) ; 6$ percent experienced PTA for 24 hours to 7 days, 36 percent experienced PTA for 7 to 30 days, and 58 percent experienced PTA for $>30$ days. PTA duration was determined with the Memory, Orientation, and Amnesia Test, which is adapted from the Galveston Orientation and Amnesia Test (GOAT) [31]. If the patient emerged from PTA before treatment at the Tampa VAMC, the PTA duration was estimated based on medical records and reports of the patient and family regarding the amount of time the patient remained confused and disoriented following the TBI. PTA duration can be estimated retrospectively with good reliability. McMillan et al. reported high correlation between prospective estimates of PTA measured by the GOAT and retrospective estimates by an independent observer [32]. By PTA duration criteria, all participants were considered to have had a moderate to severe TBI [33]. Evaluations took place a mean $\pm \mathrm{SD}$ of $25.5 \pm$ 20.3 (range $=2.4-70$ ) months postinjury.

\section{Other Raters}

The other raters were primarily female (85\%). Twothirds of the ratings were completed by parents or siblings (65\%), with the remaining completed by spouses or significant others $(27 \%)$ or friends or other family members $(9 \%)$.

\section{Measures}

\section{Key Behaviors Change Inventory}

The KBCI is a 64-item measure that was developed to assess executive, interpersonal, and emotional functioning behaviors following TBI [17]. Forty-five percent of the items are positively phrased and reverse-scored to reduce the possibility of an acquiescent response bias. Items are rated on a 4-point scale, from "False" ("Not at All True") to "Very True" and summed to create scale scores that reflect areas of difficulty typical of individuals with TBI: unawareness, inattention, impulsivity, apathy, interpersonal difficulties, communication problems, emotional adjustment, and somatic difficulties. Examples of items from each scale are presented in Table 1. Higher scores indicate more severe problems. Investigations of the measure's psychometric properties have found adequate reliability, content validity, and construct validity

Table 1.

Key Behaviors Change Inventory (KBCI) sample items.

\begin{tabular}{cc}
\hline Scale & Item: This Person ... \\
\hline Unawareness & Gets into trouble and does not \\
& understand why. \\
& Realizes errors and mistakes and \\
& tries to correct them when they $^{*}$ \\
& occur. $^{*}$
\end{tabular}

Inattention Has trouble with details.

Is able to focus on chore or task until it is finished.

Impulsivity Often acts without thinking. Is deliberate and careful. ${ }^{*}$

Apathy Shows little interest in things. Thinks about future and sets goals for him/herself.

Interpersonal Difficulties Gets into arguments easily. Gets along well with other people.*

Communication Talks too loudly or softly.

Problems

Sticks to the topic when talking with others.

Emotional Adjustment Copes poorly with stress. Seems happy most of time.

Somatic Difficulties Frequently complains of feeling tired.

Does not let normal aches and pains bother $\mathrm{him} /$ her. $^{*}$

${ }^{*}$ Reverse scored. Phrase listed is for other-rater version of KBCI. 
[17,34-35]. For example, internal consistencies of the scales range from 0.82 to 0.91 [17]. The KBCI has also been used to assess individuals with multiple sclerosis (MS) [17], memory disorders [36], and psychological and behavioral problems [30].

\section{Normative Data on Key Behaviors Change Inventory}

Comparative KBCI normative data were available from 96 individuals who did not have any medical, neurological, or mental health problems and who were asked to rate someone they knew very well (e.g., spouse, sibling, best friend). Raters were either psychology students at a local university or community-dwelling individuals who had family members with either TBI or MS. Raters were also asked to rate a "normal control," who was demographically comparable to their family member with TBI or MS. These ratings were used for normative comparisons in the current study. The ratees were an average of $36.7 \pm 15.7$ years old (mean \pm SD) and 94 percent male. The demographic characteristics of both raters and ratees were comparable to those of the study sample. Although KBCI raw scores were used for all statistical analyses, figures were plotted with normative-based $T$-scores for ease of comparing performance levels.

\section{Descriptive Data}

Patient age, sex, and injury type were obtained from clinical interview or the medical record. Measures of injury severity were obtained directly from the medical record or estimated from admission notes, progress notes, and witness reports when not clearly documented. Family raters completed a brief form indicating their age, sex, and relationship to the individual with TBI.

\section{Procedures}

At time of follow-up, each participant with a TBI and a family member or friend completed the KBCI for two time periods, one to retrospectively rate functioning prior to the TBI and the other to rate current functioning.

\section{RESULTS}

\section{Presence of Awareness Deficits}

A series of $2 \times 2$ repeated measures analyses of variance (ANOVAs) were conducted with time (pre- versus postinjury) and rater (self [patient] versus other) as the grouping factors and the raw scores on the eight KBCI scales as dependent measures. Regardless of scale, significantly greater problems were reported post- versus preinjury ( $p<0.001$, see Figure 1 for mean normative $T$ scores). Follow-up $t$-tests revealed that other raters reported greater problems on all scales postinjury $(p<$ $0.03)$. Patients' self-ratings revealed greater problems postinjury on all KBCI scales $(p<0.03)$ except the unawareness and interpersonal difficulties scales. As can

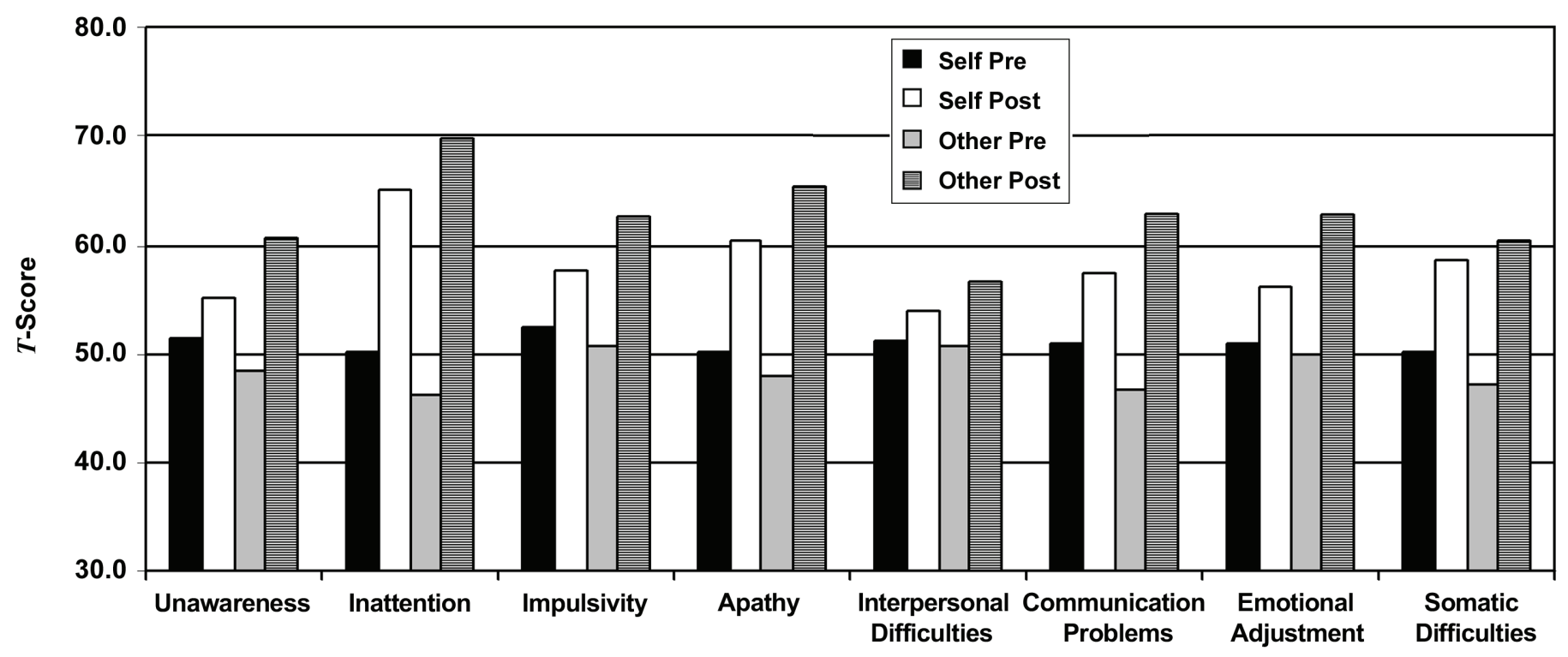

Figure 1.

Mean $T$-scores associated with each Key Behavior Change Inventory scale both pre- and postinjury for self- and other ratings. 
be seen from Figure 1, inattention was the most significant problem postinjury according to both self- and other raters. According to other raters, patients' awareness was clearly impaired following TBI, with an average $T$-score of 61. Interestingly, however, patients' self-ratings did not show a significant increase in awareness problems following TBI.

An alternative method of assessing patient's awareness problems post-TBI is examining discrepancies between self- and other ratings across KBCI scales. We calculated a composite global discrepancy score (GDS) by subtracting self-ratings from other ratings across all eight KBCI scales and then summing the differences. We then examined this GDS using a $t$-test to compare pre- versus postinjury functioning. This analysis revealed a greater GDS postinjury (mean \pm SD discrepancy of $15.62 \pm$ 35.01 ) than preinjury (mean \pm SD discrepancy of $-7.92 \pm$ $27.32 ; t_{35}=3.34, p<0.002$ ). In other words, preinjury self-other rating discrepancies on average differed by $<1$ raw score point per KBCI scale (possible discrepancy range for each scale of 0 to 24), but postinjury other ratings revealed significantly more impairment than selfratings (15.62 raw score points).

\section{Relationship Between Self-Other Global Discrepancy Score and Key Behaviors Change Inventory Unawareness Scale}

To explore the concurrent validity of the two approaches for assessing post-TBI awareness problems, we examined the correlations between the GDSs and the postinjury $\mathrm{KBCI}$ unawareness scale. As expected, the correlation between the GDSs and the other ratings was significant $(r=0.67, p<0.001)$, while the correlation for the self-ratings was not $(r=-0.12, p=0.48)$.

\section{Relationship Between Injury Variables and Postinjury Awareness Problems}

The association between post-TBI awareness problems and injury severity and time postinjury was examined. Given that the relationship between original injury severity (GCS) and post-TBI awareness problems is confounded by time postinjury, we used partial correlations to remove the confounding effect. Similarly, in the relationship between time postinjury and post-TBI awareness problems, the effect of injury severity (GCS) was removed by use of partial correlation. As can be seen in Table 2, the positive correlation between self-ratings on the KBCI unawareness scale and months postinjury indi- cates that within this sample of individuals with moderate to severe TBI, patients reported greater problems on the unawareness scale as time postinjury increased $(r=0.33)$. This result suggests that as time postinjury increased, patients showed more accurate self-awareness than patients whose injuries were more recent. Similarly, patients with less severe initial injuries were more aware of their postinjury difficulties $(r=0.42)$. In contrast, nonsignificant relationships were found between others' ratings of KBCI awareness problems and injury characteristics. Also, the self-other global discrepancy approach to assessing awareness problems was unrelated to injury characteristics, which suggests less sensitivity to awareness problems with this discrepancy approach.

\section{Prevalence of Awareness Problems Postinjury}

The preceding analyses clearly show that unawareness of problems is an important postinjury deficit in individuals with TBI. However, these analyses do not address whether these results are a universal finding in all cases of TBI or whether subgroups are present with varying levels and types of awareness problems. To address these issues, we conducted three sets of analyses: (1) concordance patterns of self-other ratings, (2) frequency of unawareness subgroups defined by $\mathrm{KBCI}$ normative data, and (3) uniformity of unawareness of problems in subgroups across different types of behavioral domains (e.g., executive problems, interpersonal interactions, and adjustment concerns).

\section{Concordance of Self-Versus Other Ratings}

Self-other discrepancy ratings were calculated for each of the eight KBCI scales for both pre- and postinjury functioning. Concordance was defined as self- and

\section{Table 2.}

Partial correlations between measures of injury severity and acuteness with measures of awareness problems. In correlations between Glasgow Coma Scale (GCS) and unawareness, effect of time postinjury is removed. In correlations between time postinjury and unawareness, effect of initial GCS is removed. Unawareness scale of Key Behaviors Change Inventory (KBCI) scores represent ratings postinjury.

\begin{tabular}{lcccc}
\hline \multirow{2}{*}{ Variable } & \multicolumn{2}{c}{ KBCI Unawareness } & & GDS \\
\cline { 2 - 3 } GCS & Self & Other & & Postinjury \\
Months Postinjury & $0.33^{\dagger}$ & -0.05 & & 0.13 \\
${ }^{*} p<0.01$. & & -0.25 \\
${ }^{\dagger} p<0.05$. \\
GDS $=$ global discrepancy score. & & \\
\hline \hline
\end{tabular}


other ratings within 3 raw score points of each other on a particular scale (two-thirds of one SD of normative mean). We calculated frequency counts for each scale to determine agreement percentage between self- and other ratings both pre- and postinjury and frequency of disagreement (i.e., self-rating of more or less impaired relative to other rating). Table 3 shows that these self-other rating concordance patterns varied broadly. Agreement percentage ranged from 56 to 83 percent on preinjury functioning, and the pattern followed either a normal distribution or a positive skew. Postinjury, however, the curve became negatively skewed because of the increase in discrepancy between self- and other ratings, i.e., others rated patients as having more problems than patients rated themselves. Postinjury agreement concordance was consistently lower than preinjury concordance, ranging from 39 to 64 percent agreement. Wilcoxon matched pairs signed rank test comparisons of pre- versus postinjury rating concordance revealed significant concordance differences on all KBCI scales except apathy, which approached significance $(p=0.06)$.

Somatic difficulties elicited the greatest agreement ( $83 \%$ agreement on preinjury ratings and $64 \%$ agreement on postinjury ratings), with the least agreement seen on apathy scores postinjury (39\% agreement). Across scales, patients' preinjury self-ratings indicated greater problems than the others' ratings did, while their postinjury selfratings indicated fewer problems than the others' ratings did (Table 3). However, unawareness subgroups were present, e.g., patients whose postinjury self-ratings were comparable to the other ratings (apparent self-awareness of problems) or those with apparent unawareness postin- jury. Some patients appeared to be even more aware of their problems than the other raters postinjury.

\section{Normatively Based Key Behaviors Change Inventory Unawareness Subgroups}

Those patients whose self-ratings suggested intact awareness (according to normative comparisons on the KBCI unawareness scale, i.e., within 1 SD of the normative mean) and whose other ratings also suggested awareness were identified as the "aware per both" subgroup ( $n=14,39 \%$ of sample). Those patients whose selfratings suggested awareness but whose other ratings suggested unawareness were identified as the "unaware per other" subgroup ( $n=10,28 \%$ of sample). Those patients whose self-ratings suggested unawareness and whose other ratings suggested awareness comprised the "unaware per self" subgroup ( $n=2,5 \%$ of sample). Finally, those patients who rated themselves as unaware and whose family member rated them as unaware were classified as the "unaware per both" subgroup $(n=10$, $28 \%$ of sample). These four subgroups did not differ significantly on education, age, GCS scores, or time postinjury $(p>0.05)$. As with the general concordance data, clear unawareness subgroups were present, the largest of which (39\% of the sample) was aware of their postinjury problems according to both themselves and others.

\section{Uniformity of Unawareness of Problems in Subgroups Across Different Behavioral Domains}

A mixed model ANOVA was conducted for each KBCI scale (except unawareness, which defined the subgroups). Between-subjects classification factors were

Table 3.

Percent concordance for self- and other ratings across Key Behaviors Change Inventory scales.

\begin{tabular}{|c|c|c|c|c|c|c|c|}
\hline \multirow{2}{*}{ Scale } & \multicolumn{3}{|c|}{ Preinjury } & \multicolumn{3}{|c|}{ Postinjury } & \multirow{2}{*}{$p$-Value ${ }^{*}$} \\
\hline & Pt $>$ Other & Pt $=$ Other & Other $>$ Pt & Pt $>$ Other & $\mathbf{P t}=$ Other & Other $>$ Pt & \\
\hline Unawareness & 31 & 56 & 14 & 17 & 44 & 39 & 0.03 \\
\hline Inattention & 25 & 67 & 8 & 19 & 44 & 36 & 0.03 \\
\hline Impulsivity & 25 & 58 & 17 & 11 & 58 & 31 & 0.05 \\
\hline Apathy & 17 & 72 & 11 & 17 & 39 & 44 & 0.06 \\
\hline Interpersonal Difficulties & 22 & 61 & 17 & 14 & 53 & 33 & 0.04 \\
\hline Somatic Difficulties & 17 & 83 & 0 & 14 & 64 & 22 & 0.04 \\
\hline
\end{tabular}


rater (self versus other) and awareness subgroup (aware per both, unaware per other, unaware per both), and the within-subjects factor was evaluation time (pre- versus postinjury). The unaware per self subgroup contained only two participants and was therefore not included in these analyses.

ANOVA results indicated a main effect for awareness subgroup on all scales $(p<0.03)$ except somatic difficulties $(p=0.12)$. Across scales, those patients in the aware per both subgroup had fewer reported problems both pre- and postinjury than patients in the unaware per other or unaware per both subgroups. A main effect for time (pre- versus postinjury) was found for all scales ( $p<$ 0.001 ), with greater problem severity reported postinjury. Finally, a main effect for rater was found only on the emotional adjustment $(p<0.01)$ and impulsivity scales $(p<0.05)$, with the others' ratings indicating that individuals with TBI were more impaired than the patients' self-ratings. Significant interactions modified these main effects, however.

Three-way interactions between rater, unawareness group, and pre- versus postinjury ratings were found for four scales: apathy $(p<0.05)$, inattention $(p<0.05)$, interpersonal difficulties $(p<0.05)$, and communication problems $(p<0.03)$. As shown in Figure 2, examining the means revealed that others' ratings indicated more problems postinjury than patients' self-ratings did for the unaware per other and unaware per both subgroups versus the aware per both subgroup. Follow-up $t$-tests of simple main effects on these four KBCI scales revealed that self- versus other ratings did not differ in either the aware per both or the unaware per both subgroups for either pre- or postinjury ratings. That is, patients who either reported normatively intact awareness on the $\mathrm{KBCI}$ or normatively problematic awareness tended to agree with the other raters across these four KBCI scales. In contrast, the unaware per other subgroup had comparable self- and other ratings on preinjury functioning (on all KBCI scales) but significantly different self- versus other ratings on postinjury functioning for the inattention, apathy, and interpersonal difficulties scales. The exception to this general pattern was the communication problems scale, where self- and other ratings of postinjury functioning differed for both the unaware per other $(p<0.04)$ and unaware per both subgroups $(p<0.01)$, with the others' ratings indicating that individuals with TBI had more communication problems than the patients' self-ratings.
Two additional KBCI scales showed similar threeway interaction patterns (Figure 2) and approached statistical significance: emotional adjustment $(p=0.07)$ and impulsivity $(p=0.09)$. However, for these two scales, we found a two-way interaction $(p<0.05)$ between time and rater such that the self- and other ratings were in agreement preinjury but the others' ratings showed greater impairment postinjury. The only scale not showing a three-way interaction pattern was somatic difficulties $(p=0.55)$. As can be seen in Figure 2, others' ratings on the somatic difficulties scale were comparable to patients' self-ratings for pre- versus postinjury functioning $(p>0.05)$.

\section{DISCUSSION}

Results from this study replicate earlier reports of selfawareness problems, as well as other cognitive and adjustment problems, following TBI. Each of the eight KBCI scales was significantly elevated postinjury compared with preinjury. However, even though others' ratings of patients with TBI were significantly higher post- versus preinjury on all scales including unawareness, the patients' self-ratings on the unawareness scale were not significantly higher than their preinjury ratings, which was the first indication of self-awareness problems following TBI.

Several other indications of self-awareness problems following TBI were found. GDSs across behavioral domains between self- and other raters were large postinjury but comparable preinjury. Further, the GDS was highly correlated with direct ratings of awareness problems by others (unawareness scale), which demonstrates convergent validity for these two methods of awareness assessment. In this sample, the overall magnitude of awareness problems was moderate ( $T$-score of 62 ) as rated by others and roughly comparable to additional behavioral disturbances common after TBI (e.g., impulsivity, apathy).

The pattern of correlations between injury severity and time postinjury with self- versus other ratings on the $\mathrm{KBCI}$ unawareness scale suggests that as time postinjury increases, individuals with TBI become more accurate in their awareness ratings. This finding is consistent with those studies reporting that concordance between patients' and families' behavioral report is initially poor but improves with time postinjury $[12,26,29]$. However, 


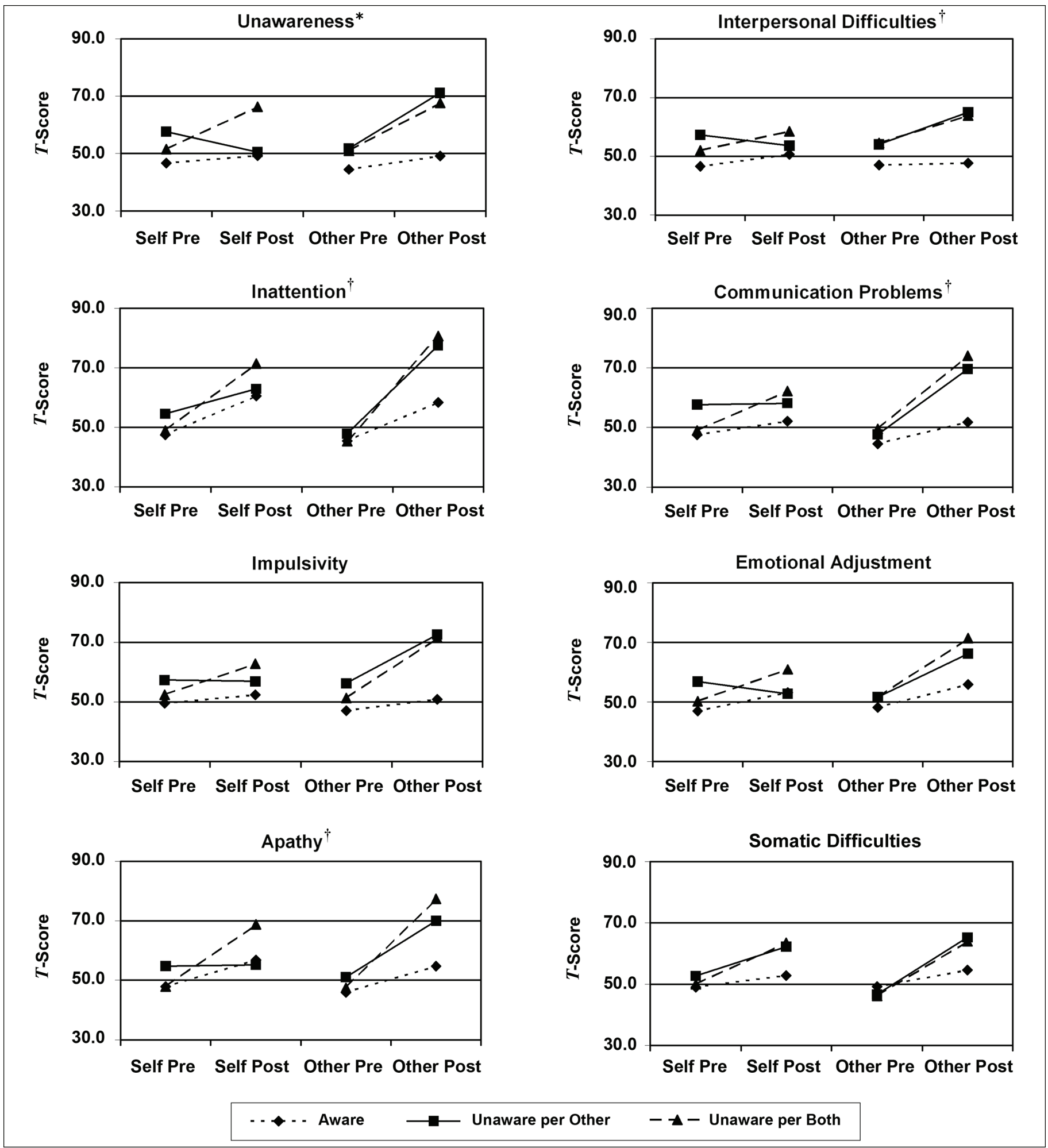

Figure 2.

Mean Key Behaviors Change Inventory by awareness group for self- (Self) vs other (Other) ratings both pre- (Pre) and postinjury (Post). Threeway interactions for emotional adjustment $(p=0.07)$ and impulsivity $(p=0.09)$ approached significance; only somatic difficulties did not show this effect $(p=0.55) .{ }^{*}$ Unawareness scale used to form awareness subgroups. ${ }^{\dagger}$ Significant three-way interactions at $p<0.01$. 
given that the others' ratings of patients' awareness problems were unrelated to time postinjury, the actual level of awareness problems likely remains relatively stable across time, at least in this postacute phase (several months to several years postinjury). Finally, we found that the less severe the original TBI (i.e., the higher the initial GCS), at least with moderate to severe TBI, the more awareness of their problems patients exhibited.

However, self-unawareness postinjury is not a universal finding in TBI. Postinjury ratings of functioning across a variety of executive, interpersonal, and adjustment domains often showed good agreement between self- and other raters. In fact, using normatively based self- versus other ratings on the KBCI unawareness scale, we found that 39 percent of the sample had no problems with awareness (aware per both). In an additional 28 percent, both patients and others reported similarly high normative levels of awareness problems (unaware per both). That is, these participants had at least enough awareness of their problems to report them as problematic. These two subgroups, representing 67 percent of the entire sample, did not differ from each other in ratings of postinjury functioning for any of the eight KBCI scales. These findings are consistent with those of Cavallo et al. [21] and Seel et al. [22]. Nevertheless, Table 3 demonstrates the shift in concordance that occurred between self- and other raters from pre- to postinjury; other raters reported significantly greater problems postinjury than self-raters did, which is another indication of at least some degree of awareness problems subsequent to TBI. Perhaps most interesting is the subgroup of patients who were in the unaware per both group ( $28 \%$ of the sample). In this subgroup, other raters saw patients as having significant problems with awareness. However, counterintuitively these individuals with TBI also had self-ratings indicative of problems with awareness. How can someone with awareness problems report having problems with awareness? This group may represent patients who have intellectual awareness of their behavioral deficits but who lack "online" awareness of when a deficit is adversely affecting their performance [37-39]. This condition has been termed emergent awareness, and about 28 percent of the sample exhibited emergent awareness. Following this group over time to determine if this type of awareness represents a transitional stage or is a stable chronic condition would be interesting.

Finally, a small percentage $(5 \%)$ of patients rated themselves as unaware but other raters saw them as aware. Family members may be in denial about their loved one's deficits or they may not be spending as much time with the patients as the caregivers in the other groups. This group requires further investigation.

Even in those individuals who were rated by others as having significant awareness problems but who were unaware of those deficits themselves (unaware per other subgroup), patients' degrees of unawareness were not uniform across behavioral domains. These individuals may fully be able to identify and accurately report their functioning in the area of somatic difficulties. This result is consistent with previous literature that found higher agreement on more external behaviors such as physical impairments than on more internal behaviors such as attention $[15,25]$. In this study, the greatest self-other discrepancies were seen on postinjury apathy scores $(38 \%$ agreement) followed closely by unawareness and inattention (each with $44 \%$ agreement).

The finding that patients are able to reliably report their preinjury status is an important one. As clinicians, assuming that anosognosic patients are unable to reliably report their medical or psychiatric history or their level of premorbid functioning is often tempting. Our data suggest that even patients with significant awareness problems are capable of reporting on their preinjury status, at least in this nonacute state (an average of 23 months postinjury). These findings may not generalize to more acute patients and contrast with a previous report that overall, individuals with TBI tend to overestimate their preinjury competence (relative to reports of informants) at least on certain items [40].

This study has several limitations. First, the sample size was relatively small $(n=36)$, which resulted in small unawareness subgroups for the follow-up analyses and limited the power to detect group differences. Nevertheless, significant group differences were found for most KBCI scales, both in the full sample and in the unawareness subgroups. In addition, we did not correct for multiple comparisons, thereby increasing the likelihood of spurious results. Another limitation is that ratings of preinjury functioning were done retrospectively. Therefore, postinjury factors may have distorted ratings of preinjury functioning. However, we should note that self- and other ratings of preinjury functioning were comparable for all KBCI scales and for normative levels of functioning, both in the full sample and the subgroups. This result suggests that the retrospective rating of preinjury functioning was an effective and reliable approach in this sample. Finally, 
given the possible link between awareness and psychiatric status (i.e., depression) [41], future studies should include analysis of psychiatric factors and their interaction with awareness problems.

\section{CONCLUSIONS}

Results of this study suggest that unawareness may not be universal following TBI nor is it evidenced in a uniform manner across behavioral domains. Study findings suggest that patients are generally good historians regarding their preinjury functioning but collateral sources provide valuable information during assessment of postinjury functioning. Nonetheless, self-other discrepancies depend upon characteristics of the patient and the behavior in question, and therefore, awareness assessment must not rely solely on self-other discrepancies. Furthermore, though awareness may improve somewhat in the initial months postinjury, some individuals with TBI continue to lack awareness of problems even several years later. Therefore, awareness problems should be evaluated in chronic, as well as acute, injury states because patients who are unaware will be variably able to participate successfully in rehabilitation, function in the environment, and interact socially. Awareness assessment may be complicated by the different ways in which a given patient may manifest unawareness, which range from complete unawareness to emergent awareness. Relying on behavioral awareness assessment, asking significant others about behavior, and asking significant others specifically about awareness may all be methods for gaining a more comprehensive assessment of awareness in patients following TBI.

\section{ACKNOWLEDGMENTS}

The views expressed in this article are those of the authors and do not necessarily represent the views of the VA.

This material is based on work supported by the VA, Veterans Health Administration. Further support was provided by the DVBIC, the Uniformed Services University of the Health Sciences, Bethesda, Maryland, the James A. Haley Veterans' Hospital where the study was completed, and a grant from the Henry M. Jackson Foun- dation for the Advancement of Military Medicine (MDA 905-03-2-0003).

The authors have declared that no competing interests exist.

\section{REFERENCES}

1. Stuss DT. Disturbance of self-awareness after frontal system damage. In: Prigatano GP, Schachter DL, editors. Awareness of deficit after brain injury: Clinical and theoretical issues. New York (NY): Oxford University Press; 1991. p. 63-83.

2. Sherer M, Bergloff P, Levin E, High WM Jr, Oden KE, Nick TG. Impaired awareness and employment outcome after traumatic brain injury. J Head Trauma Rehabil. 1998; 13(5):52-61. [PMID: 9753535]

3. Prigatano GP. Disturbance of self-awareness of deficit after traumatic brain injury. In: G. Prigatano GP, Schachter DL, editors. Awareness of deficit after brain injury: Clinical and theoretical issues. New York (NY): Oxford University Press; 1991. p. 111-26.

4. Trudel TM, Tryon WW, Purdum CM. Awareness of disability and long-term outcome after traumatic brain injury. Rehabil Psychol. 1998;43(4):267-81.

5. Prigatano GP, Altman IM, O’Brien KP. Behavioral limitations that traumatic-brain-injured patients tend to underestimate. Clin Neuropsychol. 1990;4(2):163-76.

6. Prigatano GP, Klonoff PS. A clinician's rating scale for evaluating impaired self-awareness and denial of disability after brain injury. Clin Neuropsychol. 1998;12(1):56-67.

7. Sunderland A, Harris JE, Baddeley AD. Do laboratory tests predict everyday memory? A neuropsychological study. J Verb Learning Verb Behav. 1983;22:341-57.

8. Sunderland A, Harris JE, Baddeley AD. Assessing everyday memory after severe head injury. In: Harris JE, Morris PE, editors. Everyday memory: Actions and absent-mindedness. London (England): Academic Press; 1984. p. 191-206.

9. Bogod NM, Mateer CA, MacDonald SW. Self-awareness after traumatic brain injury: A comparison of measures and their relationship to executive functions. J Int Neuropsychol Soc. 2003;9(3):450-58. [PMID: 12666769]

10. Malec JF, Machulda MM, Moessner AM. Differing problem perceptions of staff, survivors, and significant others after brain injury. J Head Trauma Rehabil. 1997;12(3):1-13.

11. Prigatano GP, Altman IM. Impaired awareness of behavioral limitations after traumatic brain injury. Arch Phys Med Rehabil. 1990;71(13):1058-64. [PMID: 2256806]

12. Lanham RA Jr, Weissenburger JE, Schwab KA, Rosner MM. A longitudinal investigation of the concordance between individuals with traumatic brain injury and family 
or friend ratings on the Katz adjustment scale. J Head Trauma Rehabil. 2000;15(5):1123-38. [PMID: 10970933]

13. McKinlay WW, Brooks DN. Methodological problems in assessing psychosocial recovery following severe head injury. J Clin Neuropsychol. 1984;6(1):87-99. [PMID: 6699188]

14. McGlynn SM, Schacter DL. Unawareness of deficits in neuropsychological syndromes. J Clin Exp Neuropsychol. 1989;11(2):143-205. [PMID: 2647781]

15. Sherer M, Boake C, Levin E, Silver BV, Ringholz G, High WM Jr. Characteristics of impaired awareness after traumatic brain injury. J Int Neuropsychol Soc. 1998;4(4):380-87. [PMID: 9656611

16. Fleming JM, Strong J, Ashton R. Self-awareness of deficits in adults with traumatic brain injury: How best to measure? Brain Inj. 1996;10(1):1-15. [PMID: 8680388]

17. Kolitz BP, Vanderploeg RD, Curtiss G. Development of the Key Behaviors Change Inventory (KBCI): A traumatic brain injury behavioral outcome instrument. Arch Phys Med Rehabil. 2003;84(2):277-84. [PMID: 12601661]

18. Borgaro SR, Prigatano GP. Modification of the Patient Competency Rating Scale for use on an acute neurorehabilitation unit: The PCRS-NR. Brain Inj. 2003;17(10):847-53. [PMID: 12963551]

19. Sherer M, Bergloff P, Boake C, High WM Jr, Levin E. The Awareness Questionnaire: Factor structure and internal consistency. Brain Inj. 1998;12(1):63-68. [PMID: 9483338]

20. Sherer M, Hart T, Nick TG. Measurement of impaired selfawareness after traumatic brain injury: A comparison of the Patient Competency Rating Scale and the Awareness Questionnaire. Brain Inj. 2003;17(1):25-37. [PMID: 12519645]

21. Cavallo MM, Kay T, Ezrachi O. Problems and changes after traumatic brain injury: Differing perceptions within and between families. Brain Inj. 1992;6(4):327-35.

[PMID: 1638266]

22. Seel RT, Kreutzer JS, Sander AM. Concordance of patients' and family members' ratings of neurobehavioral functioning after traumatic brain injury. Arch Phys Med Rehabil. 1997;78(11):1254-59. [PMID: 9365357]

23. Pagulayan KF, Temkin NR, Machamer JE, Dikmen SS. The measurement and magnitude of awareness difficulties after traumatic brain injury: A longitudinal study. J Int Neuropsychol Soc. 2007;13(4):561-70. [PMID: 17521477]

24. Dywan J, Segalowitz SJ. Self- and family ratings of adaptive behavior after traumatic brain injury: Psychometric scores and frontally generated ERPs. J Head Trauma Rehabil. 1996;11(2):79-95.

25. Sbordone RJ, Seyranian GD, Ruff RM. Are the subjective complaints of traumatically brain injured patients reliable? Brain Inj. 1998;12(6):505-15. [PMID: 9638327]

26. Powell JM, Machamer JE, Temkin NR, Dikmen SS. Selfreport of extent of recovery and barriers to recovery after traumatic brain injury: A longitudinal study. Arch Phys Med Rehabil. 2001;82(8):1025-30. [PMID: 11494180]

27. Fischer S, Trexler LE, Gauggel S. Awareness of activity limitations and prediction of performance in patients with brain injuries and orthopedic disorders. J Int Neuropsychol Soc. 2004;10(2):190-99. [PMID: 15012839]

28. Sander AM, Seel RT, Kreutzer JS, Hall KM, High WM Jr, Rosenthal M. Agreement between persons with traumatic brain injury and their relatives regarding psychosocial outcome using the Community Integration Questionnaire. Arch Phys Med Rehabil. 1997;78(4):353-57.

[PMID: 9111453]

29. O'Keefe F, Dockree P, Moloney P, Carton S, Robertson IH. Awareness of deficits in traumatic brain injury: A multidimensional approach to assessing metacognitive knowledge and online-awareness. J Int Neuropsychol Soc. 2007;13(1): 38-49. [PMID: 17166302]

30. Teasdale G, Jennett B. Assessment of coma and impaired consciousness. A practical scale. Lancet. 1974;2(7872):81-84. [PMID: 4136544]

31. Levin HS, O'Donnell VM, Grossman RG. The Galveston Orientation and Amnesia Test. A practical scale to assess cognition after head injury. J Nerv Ment Dis. 1979;167(11): 675-84. [PMID: 501342]

32. McMillan TM, Jongen EL, Greenwood RJ. Assessment of post-traumatic amnesia after severe closed head injury: Retrospective or prospective? J Neurol Neurosurg Psychiatry. 1996;60(4):422-27. [PMID: 8774408]

33. Kay T, Harrington DE, Adams R, Anderson T, Berrol S, Cicerone K, Dahlberg C, Gerber D, Goka R, Harley P, Hilt J, Horn L, Lehmkuhl D, Malec J. Definition of mild traumatic brain injury. J Head Trauma Rehabil. 1993;8(3):86-87.

34. Duchnick JD, Vanderploeg RD, Curtiss G. Sensitivity of the KBCI to emotional and behavioral problems [abstract]. Clin Neuropsychol. 2002;16:209.

35. Kolitz BP. Development of the Key Behaviors Change Inventory (KBCI), a TBI behavioral outcome assessment instrument [dissertation]. Tampa (FL): University of South Florida; 2000.

36. Belanger HG, Brown LM, Crowell TA, Vanderploeg RD, Curtiss G. The Key Behaviors Change Inventory and executive functioning in an elderly clinic sample. Clin Neuropsychol. 2002;16(3):251-57. [PMID: 12607138]

37. Barco PP, Crosson B, Bolesta MM, Werts D, Stout R. Training awareness and compensation in postacute head injury rehabilitation. In: Kreutzer JS, Wehman P, editors. Cognitive rehabilitation for persons with traumatic brain injury: A functional approach. Baltimore (MD): Paul H. Brookes; 1991. p. 129-46.

38. Crosson B. Application of neuropsychological assessment results. In: Vanderploeg RD, editor. Clinician's guide to 
neuropsychological assessment. Mahwah (NJ): Lawrence Erlbaum; 2000. p. 195-244.

39. Crosson B, Barco PP, Velozo CA, Bolesta MM, Cooper PV, Werts D, Brobeck TC. Awareness and compensation in post-acute head injury rehabilitation. J Head Trauma Rehabil. 1989;4:46-54.

40. Leathem JM, Murphy LJ, Flett RA. Self- and informant-ratings on the Patient Competency Rating Scale in patients with traumatic brain injury. J Clin Exp Neuropsychol. 1998; 20(5):694-705. [PMID: 10079045]

41. Malec JF, Testa JA, Rush BK, Brown AW, Moessner AM. Self-assessment of impairment, impaired self-awareness, and depression after traumatic brain injury. J Head Trauma Rehabil. 2007;22(3):156-66. [PMID: 17510591]

Submitted for publication December 19, 2006. Accepted in revised form July 25, 2007. 